\title{
The Effect of Lateral Guiding Mechanism on Noise Characteristics in Semiconductor Lasers
}

\author{
ABBAS ZARIFKAR ${ }^{1}$, AFSANEH FALAHATPISHEH ${ }^{2}$ \\ ${ }^{1}$ Department of Electrical Engineering, ${ }^{2}$ Switch Group \\ ${ }^{1}$ Saveh Azad University, ${ }^{2}$ Iran Telecommunication Research Center \\ ${ }^{1}$ Felestin Square, P.O. Box: 39187-366,Saveh, ${ }^{2}$ North Kargar St., P.O.Box:14155-3961,Tehran
} IRAN

Abstract: A comparison between intensity and frequency noise spectra and also the line shapes of gainguided, weakly-index-guided, and strongly-index-guided semiconductor lasers are made using numerical solution of the field and carrier density rate equations including spontaneous emission noise.

Key-Words: Spatio-Temporal Analysis, Intensity Noise, Lineshape, Semiconductor Laser, Numerical Simulation.

\section{Introduction}

Usually, for the calculation of noise characteristics in semiconductor lasers, the spatially independent Langevin rate equations are used. This approach offers the advantage of reducing a multidimensional set of partial differential equations to a one-dimensional set of ordinary differential equations. In such treatments the structural features of laser in the lateral direction is usually not included. In this paper, the rate equations for the optical field and the carrier density are solved numerically and the intensity and frequency noise spectra and also the line shape of laser are calculated from the numerical results. Based on these results, a comparison between gain-guided, weakly-index-guided, and strongly-index-guided semiconductor lasers is made.

\section{The model Equations}

The starting point for the spatio-temporal analysis of noise in semiconductor lasers is use of the rate equations for the lateral field distribution $E(x, t)$ and the carrier density $N(x, t)$ including noise, which are given by [1]-[8]:

$$
\begin{aligned}
\frac{\partial E(x, t)}{\partial t}= & j \frac{\Gamma \omega \mu_{a}}{\mu^{2}} \Delta \mu_{e f f}(x) E(x, t)+ \\
& \frac{1-j \beta_{c}}{2}\left[A\left(N-N_{0}\right)-\gamma\right] E(x, t)+ \\
& \frac{j c^{2}}{2 \omega \mu^{2}} \frac{\partial^{2} E(x, t)}{\partial x^{2}}+\chi(x, t)
\end{aligned}
$$

$$
\begin{aligned}
\frac{\partial N(x, t)}{\partial t}= & \frac{J(x)}{e d}-N(x, t) / \tau_{s}+D \frac{\partial^{2} N(x, t)}{\partial x^{2}} \\
& -A\left(N-N_{0}\right)|E|^{2}-\left(E^{*} \chi+E \chi^{*}\right)
\end{aligned}
$$

where $\Gamma$ is the transverse confinement factor, $\omega$ is the optical frequency, $\mu_{a}$ is the refractive index of the active layer, $\mu$ is the effective index, $\Delta \mu_{e f f}(x)$ is the effective lateral index variation, $\beta_{c}$ is the linewidth enhancement factor, $A$ is the gain coefficient, $N_{0}$ is the carrier density at transparency, $\gamma$ is the distributed loss, $c$ is the velocity of light, $J(x)$ is the current density distribution, $e$ is the electron charge, $d$ is the active layer thickness, $\tau_{s}$ is the carrier lifetime, and $D$ is the diffusion constant.

The effective built-in index variation $\Delta \mu_{\text {eff }}(x)$ in Eq. (1) is a consequence of different lateral environments experienced by the waveguide mode inside and outside the emission region. It can be approximated by [9]:

$$
\Delta \mu_{\text {eff }}(x)=\frac{\Delta \mu_{L}}{1+\exp \left(\frac{|x|-W / 2}{\sigma}\right)}
$$

where $\Delta \mu_{L}$ is the index step, $W$ is the stripe width, and $\sigma$ is the width of the transition region over which the index difference varies from $\Delta \mu_{L}$ to 0 in the vicinity of the emitter boundary at $|x|=W / 2$. For gain-guided, weakly-index-guided, and strongly -index-guided semiconductor lasers the value of 
$\Delta \mu_{L}$ is assumed to be $0,0.01$, and 0.2 , respectively [10]-[11].

The random spontaneous emission process is modeled by a noise term $\chi(x, t)$ taken as a complex Gaussian white noise of zero mean value and correlation given by [12]-[17]:

$\left\langle\chi(x, t) \chi\left(x^{\prime}, t^{\prime}\right)\right\rangle=\frac{\beta_{s p} B \bar{N}^{2}}{L d} \delta\left(x-x^{\prime}\right) \delta\left(t-t^{\prime}\right)$

where $\bar{N}$ is the steady state average value of the carrier density, $B$ is the spontaneous recombination coefficient, $L$ is the cavity length, and $\beta_{s p}$ is a coefficient determined by the lateral guiding mechanism of laser.

The current density $J(x)$ that appears in Eq. (2) is the distribution of current entering the active region, which has therefore undergone some current spreading in the cladding layer. The exact determination of $J(x)$ requires solution of twodimensional diffusion equation, but for gain-guided and weakly-index-guided lasers we assume that the $J(x)$ has the form [9], [18]-[19]:

$J(x)= \begin{cases}J_{0} & |x| \leq W / 2 \\ J_{0} e^{-K(|x|-W / 2)} & |x|>W / 2\end{cases}$

where $J_{0}$ is a constant value and $K$ is a parameter that determines the shape of the distribution. For strongly-index-guided lasers the current is taken to be zero outside the stripe [20].

\section{Results of Simulation}

For solving the model Eqs. (1) and (2), first we must obtain the steady state average values by considering $\partial / \partial t=0$. This results in a set of ordinary differential equations of $x$ that is solved by finite difference method. We assume that the $x$ derivatives of the electric field and the carrier density are zero at boundaries [21].

The typical parameter values used has been given in [1], [3], and [5].

The results of the steady state solution for spatial variations of the carrier density and field intensity are shown in Figs (1) and (2), respectively.

Using steady state average values we can include noise terms in Eqs. (1) and (2) and solve these equations numerically by hopscotch method. In this method, after discretization in the $x$ direction, the grid points are divided into even and odd points. There are four computational cycles to perform in order to advance the solution to the next time level.

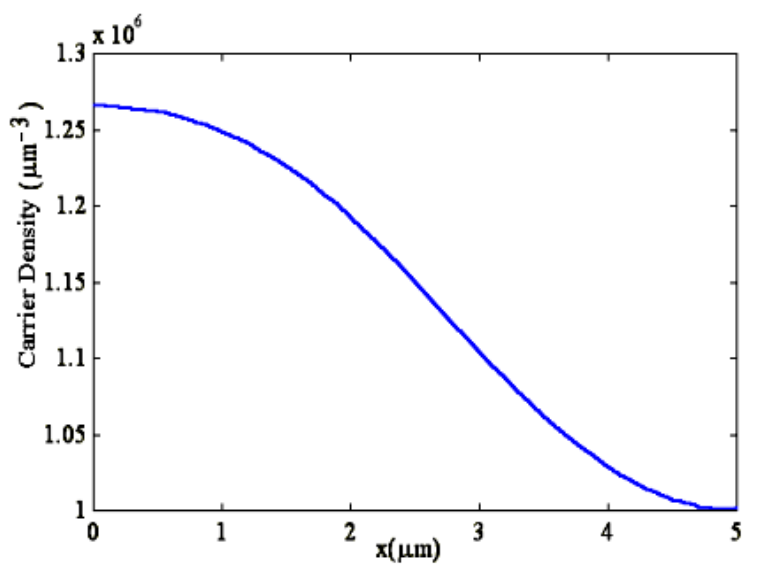

Fig 1: the carrier density distribution in the lateral direction.

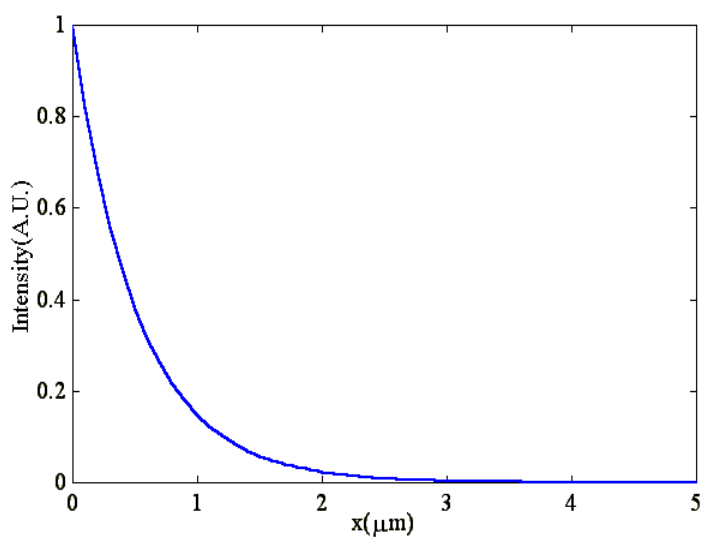

Fig 2: field intensity distribution in the lateral direction.

In the first cycle, only the even points are advanced by $\Delta t$ explicitly, which is trivial. In the second cycle, only the odd points are advanced by $\Delta t$ implicitly. In the next two cycles, the process is reversed. In the third cycle all odd points are advanced first explicitly, and then in the fourth cycle all even points are computed implicitly.

For numerical simulation of noise source we use the following relation:

$\chi(x, t)=\sqrt{\frac{\beta_{s p} B \bar{N}^{2}}{2 L d}} G_{t} G_{x}(1+j) \frac{1}{\sqrt{\Delta t}} \frac{1}{\sqrt{\Delta x}}$

where $G_{t}$ and $G_{x}$ are Gaussian random variables with zero mean and variance equal to1.

From the numerical results, we calculated the relative intensity noise (RIN), Frequency Noise Spectra (FNS), and the laser field spectra for the three types.

Figs. 3(a)-3(c) show the RIN for gain-guided, weakly-index-guided, and strongly-index-guided semiconductor lasers, respectively. It is evident from Fig. 3 that as the lateral guiding mechanism is 
changed from gain-guiding to weakly-index-guiding and then to strongly-index-guiding, the intensity noise behavior is modified.

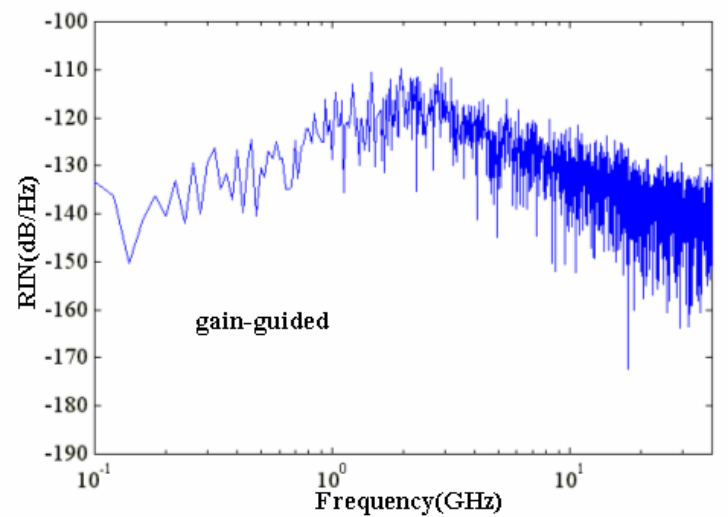

(a)

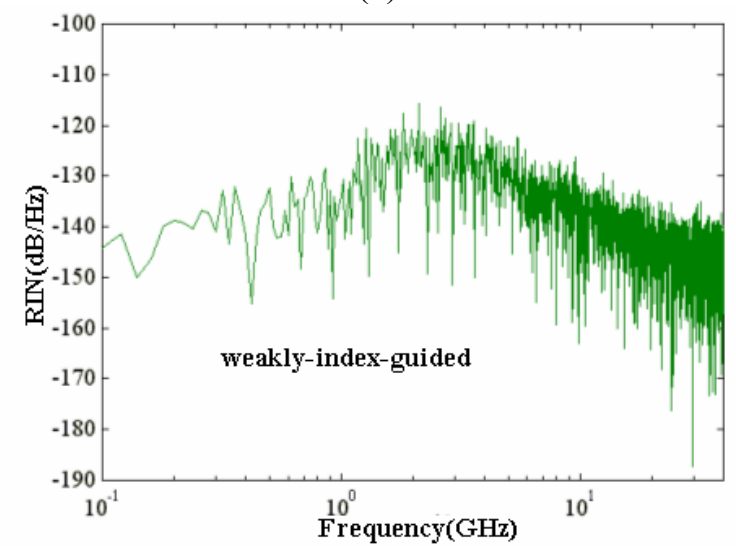

(b)

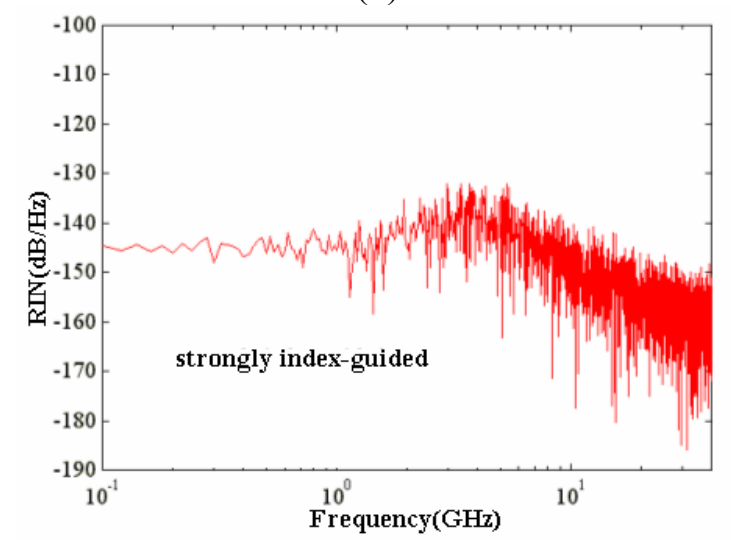

(c)

Fig. 3 Relative intensity noise spectra for (a) gainguided, (b) weakly-index-guided, and (c) strongly-index-guided semiconductor lasers.

The lateral confinement machanism of laser also affects the frequency noise characteristics. Figs. 4(a)-4(c) show the FNS for three types of semiconductor lasers.

The line width of laser is a manifestation of the laser frequency noise. In Figs. 5(a)-5(c) the field spectra for the three laser structures are shown. As seen in these figures the frequency noise of indexguided laser is lower and the line width of it is narrower than that of gain-guided lasers. This is due to the superior confinment of optical mode in these structures. The calculations also show that the frequency noise spectrum qualitatively has a bahavior like the ralative intensity noise spectrum.

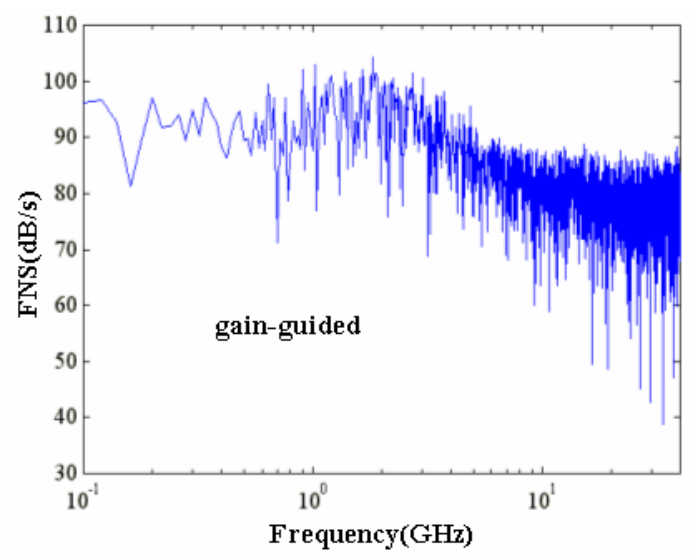

(a)

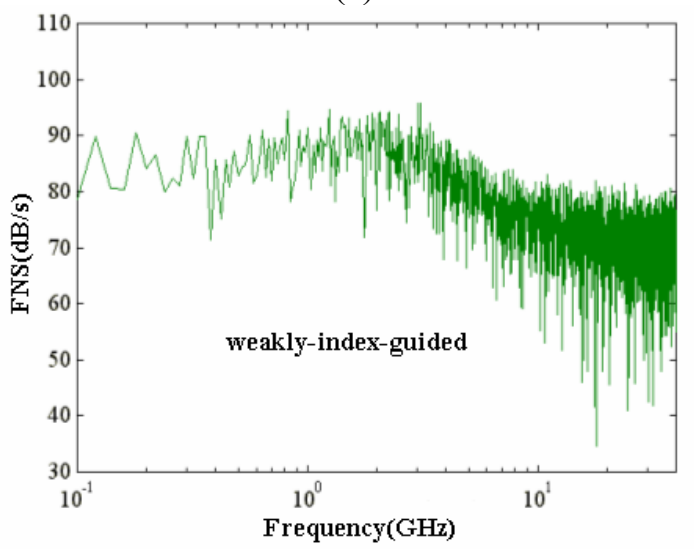

(b)

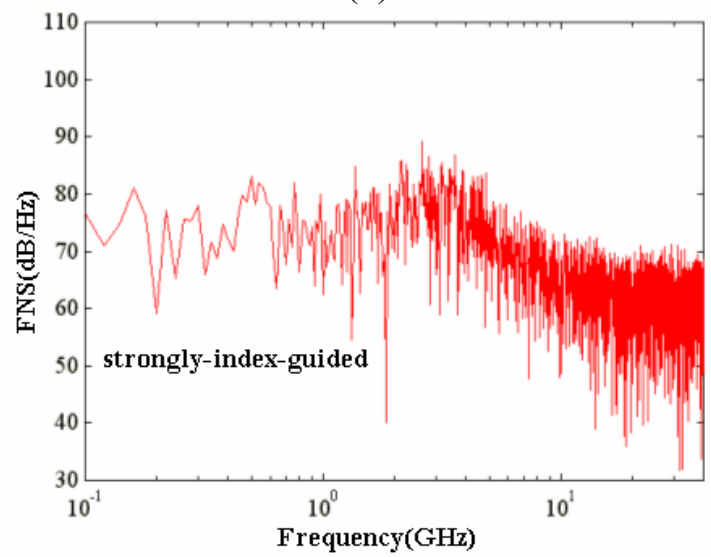

(c)

Fig. 4 Frequency noise spectra for (a) gain-guided, (b) weakly-index-guided, and (c) stronglyindex-guided semiconductor lasers. 


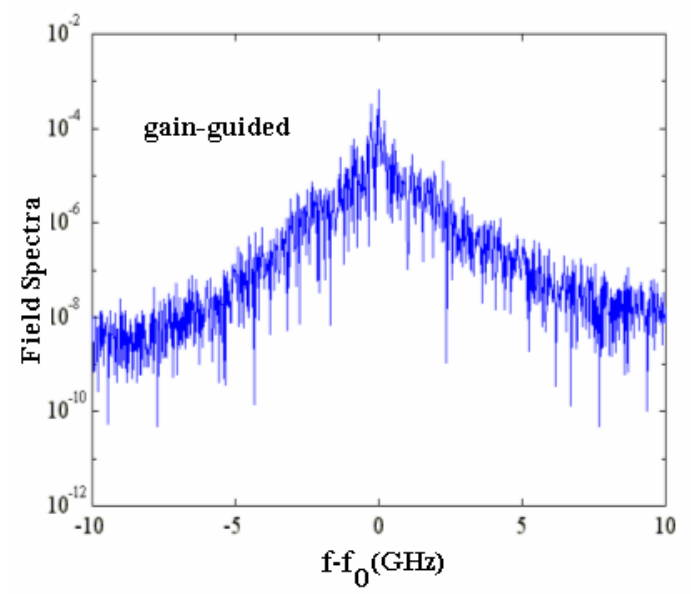

(a)

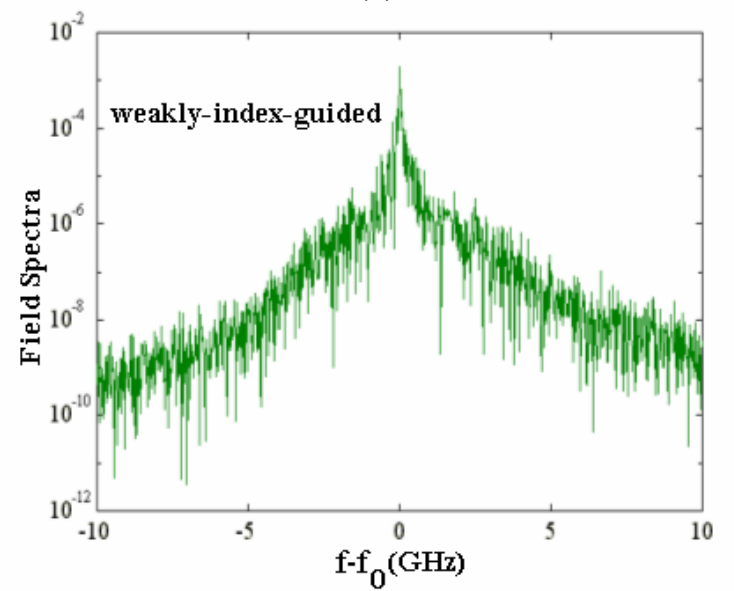

(b)

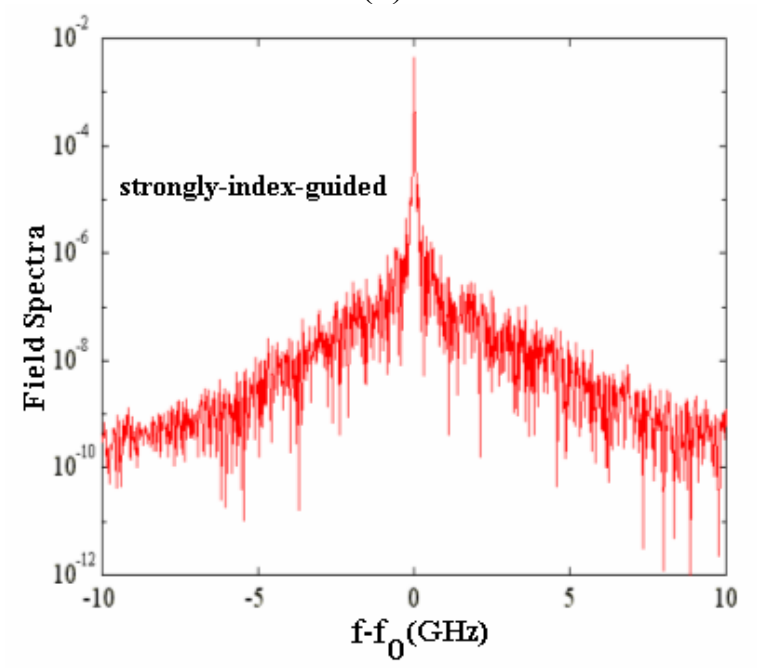

(c)

Fig. 5 Field spectra for (a) gain-guided, (b) weaklyindex-guided, and (c) strongly-index-guided semiconductor lasers.

\section{Conclusion}

Space-time analysis of semiconductor laser noise was done in order to consider the structural features of laser in the lateral direction. Index-guided lasers have better noise characteristics respect to gainguided lasers and their intensity noise, frequency noise, and line width is lower. Further, by increasing the lateral index step, the noise performance will be improved.

References:

[1] J. Martin-Regalado, S. Balle and N. B. Abraham, Spatio-Temporal Dynamics of Gain-Guided Semiconductor Laser Arrays, IEEE J. Quantum Electron., Vol. 32, No. 2, 1996, pp. 257-276.

[2] J. Martin-Regalado, S. Balle and N. B. Abraham, Modelling Spatio-Temporal Dynamics of GainGuided Multistripe and Broad-Area Lasers, IEEE Proc.-Optoelectron., Vol. 143, No. 1, 1996, pp. 17-23.

[3] S. Fernandez Casares, S. Balle and P. Menendez-Valdes, Mode Beating and Spontaneous Emission Noise Effects in a Variable-Waveguide Model for the Dynamics of Gain-Guided Semiconductor Laser Arrays, IEEE J. Quantum Electron., Vol. 30, No. 11, 1994, pp. 2449-2457.

[4] H. Adachihara, O. Hess, R. Indik and J. V. Moloney, Semiconductor Laser Array Dynamics: Numerical Simulations on Multistripe IndexGuided Lasers, J. Optical Society of America B, Vol. 10, No. 3, 1993, pp. 496-506.

[5] L. Rahman and H. G. Winful, Nonlinear Dynamics of Semiconductor Laser Arrays: A Mean Field Model, IEEE J. Quantum Electron., Vol. 30, No. 6, 1994, pp. 1405-1415.

[6] P. K. Jakobsen, J. V. Moloney, A. C. Newell and R. Indik, Space-Time Dynamics of Wide-GainSection Lasers, Phys. Rev. A, Vol. 45, No. 11, 1992, pp. 8129-8137.

[7] G. P. Agrawal, Lateral Analysis of Quasi-IndexGuided Injection Lasers: Transition from Gain to Index Guiding, J. Lightwave Technol., Vol. LT2, 1984, pp. 537-543.

[8] S. F. Yu, R. G. S. Plumb, L. M. Zhang, M. C. Newell and J. E. Carroll, Large Signal Dynamic Behavior of Distributed-Feedback Lasers Including Lateral Effects, IEEE J. Quantum Electron., Vol. 30, No. 8, 1994, pp. 1740-1750.

[9] R. J. Lang, A. G. Larsson and J. G. Cody, Lateral Modes of Broad Area Semiconductor Lasers: Theory and Experiment, IEEE $J$. 
Quantum Electron., Vol. 27, No. 3, 1991, pp. 312-320,

[10] G. P. Agrawal, Fast-Fourier-Transform based Beam-Propagation Model for Stripe-Geometry Semiconductor Lasers: Inclusion of Axial Effects, J. Appl. Phys., Vol. 56, No. 11, 1984, pp. 3100-3109.

[11] G. P. Agrawal, Lateral-Mode Analysis of GainGuided and Index-Guided Semiconductor Laser Arrays, J. Appl. Phys., Vol. 58, No. 8, 1985, pp. 2922-2931.

[12] B. Tromborg, H. Olesen and X. Pan, Theory of Linewidth for Multielectrode Laser Diodes with Spatially Distributed Noise Sources, IEEE J. Quantum Electron., Vol. 27, No. 2, 1991, pp. 178-192.

[13] B. Tromborg, H. E. Lassen and H. Olesen, Traveling Wave Analysis of Semiconductor Lasers: Modulation Responses, Mode Stability and Quantum Mechanical Treatment of Noise Spectra, IEEE J. Quantum Electron., Vol. 30, No. 4, 1994, pp. 939-956.

[14] G. H. Duan, P. Gallion and G. P. Agrawal, Dynamic and Noise Properties of Tunable Multielectrode Semiconductor Lasers Including Spatial Hole Burning and Nonlinear Gain, IEEE J. Quantum Electron., Vol. 29, No. 3, 1993, pp. 844-855.

[15] J. L. Vey and P. Gallion, Semiclassical Model of Semiconductor Laser Noise and Amplitude Noise Squeezing-Part I: Description and Application to Fabry-Perot Laser, IEEE J. Quantum Electron., Vol. 33, No. 11, 1997, pp. 2097-2104.

[16] M. Homar, J. V. Moloney and M. San Miguel, Traveling Wave Model of a Multimode FabryPerot Laser in Free Running and External Cavity Configurations, IEEE J. Quantum Electron., Vol. 32, No. 3, 1996, pp. 553-566.

[17] D. D. Marcenac and J. E. Carroll, Modeling of Intensity Noise Including Squeezing in DFB and Fabry-Perot Semiconductor Laser Diodes, IEEE J. Quantum Electron., Vol. 30, No. 9, 1994, pp. 2064-2072.

[18] S. S. Wang and H. G. Winful, Propagation Model for the Dynamics of Gain-Guided Semiconductor Laser Arrays, J. Appl. Phys., Vol. 73, No. 1, 1993, pp. 462-464.

[19] S. L. Chuang, Physics of Optoelectronic Devices, John Wiley \& Sons, Inc., 1995.

[20] G. P. Agrawal and N. K. Dutta, Semiconductor Lasers, 2nd ed., New York:Van Nostrand Reinhold, 1993.
[21] S. F. Yu, A Quasi-Three-Dimentional LargeSignal Dynamic Model of Distributed Feedback Lasers, IEEE J. Quantum Electron., Vol. 32, No. 3, 1996, pp. 424-432. 